Research Article

\title{
Association of Postoperative Neutrophil Lymphocyte Ratio (NLR) and Monocyte Lymphocyte Ratio (MLR) with the Presence of Osteoporosis in Japanese Patients after Hip Fracture Surgery: A Retrospective Cohort Study
}

\author{
Hirofumi Bekki $\mathbb{D},{ }^{1,2}$ Takeshi Arizono, ${ }^{1}$ Daiki Hama, ${ }^{1}$ Akihiko Inokuchi, ${ }^{1}$ \\ Takahiro Hamada, ${ }^{1}$ and Ryuta Imamura ${ }^{1}$ \\ ${ }^{1}$ Department of Orthopaedic Surgery, Kyushu Central Hospital of the Mutual Aid Association of Public School Teachers, \\ Fukuoka, Japan \\ ${ }^{2}$ Department of Orthopaedic Surgery, National Kyushu Medical Center, Fukuoka, Japan \\ Correspondence should be addressed to Hirofumi Bekki; hbekki@kyushu-ctr-hsp.com
}

Received 24 March 2021; Revised 18 June 2021; Accepted 30 August 2021; Published 15 September 2021

Academic Editor: Rimesh Pal

Copyright (c) 2021 Hirofumi Bekki et al. This is an open access article distributed under the Creative Commons Attribution License, which permits unrestricted use, distribution, and reproduction in any medium, provided the original work is properly cited.

Background. The diagnosis of osteoporosis is based on bone mineral density measurements expressed as a percentage of the young adult mean (YAM) in Japan. Osteoporosis is defined as YAM $<70 \%$, and intervention is recommended at this cutoff. Because osteoporosis has a strong association with systemic metabolic disorders, we postulated that patients with $\mathrm{YAM}<70 \%$ had higher inflammatory biomarker concentrations owing to the higher systemic stress compared with YAM $>70 \%$. Method. We retrospectively reviewed 94 patients with low-trauma hip fractures. Blood examinations were performed on postoperative day (POD) 1 and POD 7. We used neutrophil lymphocyte ratio (NLR) and monocyte lymphocyte ratio (MLR) to evaluate postoperative recovery. After dividing the 94 patients into two groups according to a YAM cutoff of $70 \%$, we compared the differences in NLR and MLR. Results. On POD 1, patients with YAM $>70 \%$ had a median NLR of 5.7 and a median MLR of 0.66 , which were significantly lower than for patients with YAM $<70 \%$ ( 8.8 and 0.9 , respectively). Similarly, on POD 7 , patients with YAM $>70 \%$ had a median NLR of 2.0 and a median MLR of 0.31 , which were significantly lower than for patients with YAM $<70 \%$ (3.5 and 0.43 , respectively). Conclusion. A YAM cutoff of $70 \%$ is an appropriate intervention threshold regarding postoperative recovery after hip fracture surgery. Mini-Abstract. Patients with YAM $>70 \%$ showed lower NLR and MLR on POD 1 and POD 7. A YAM cuffoff of $70 \%$ is an appropriate intervention threshold regarding postoperative recovery after hip fracture surgery.

\section{Introduction}

In Japan, the criteria for diagnosing osteoporosis prepared by the Japanese Society for Bone and Mineral Research are based on bone mineral density (BMD) measurements expressed as a percentage of the young adult mean (YAM) for women [1]. Osteoporosis is defined as $<70 \%$ of the young adult mean percentage (\%YAM) for the mean BMD in healthy young people for the lumbar spine or femoral neck
[2]. Intervention is recommended at a YAM of $70 \%$ ( $T$ score: -2.7 standard deviation) at the femoral neck [3].

Because hip fractures are associated with high morbidity and mortality, patients require emergency surgical care [4]. Considering that osteoporosis is related to systemic metabolic disorders, such as hypertension, diabetes, and pulmonary and renal dysfunction [5-8], patients with osteoporosis may undergo serious stress after surgery owing to the operative invasiveness. The purpose of this study was to confirm the 
hypothesis that a YAM cutoff of 70\% is appropriate regarding postoperative recovery for hip fractures.

\section{Materials and Methods}

2.1. Patients. In this retrospective cohort study, we reviewed 94 patients with low-trauma hip fractures (45 with trochanteric fractures, 49 with femoral neck fractures) treated at a single medical institution in Japan for 9 months from April 2020. We performed bipolar hip arthroplasty (BHA) or open reduction and internal fixation (ORIF) for hip fractures and ORIF for trochanteric fractures. YAM in the nonfractured femoral neck was measured by using the Horizon DXA System bone densitometer (Hologic Inc., Marlborough, MA). This was because the femoral neck is a consistently significant predictor of hip fractures, and the discriminant power was better than that measured at the lumbar spine [9].

Patients comprised 76 women and 18 men with a median age of 88 years (range, $61-105$ years). The median YAM was $54 \%(47 \%-67 \%) ; 77$ patients had a YAM $<70 \%$, and 17 patients had a YAM $>70 \%$. The median body mass index (BMI) was $19.8 \mathrm{~kg} / \mathrm{m}^{2}$ (range, $12.5-30 \mathrm{~kg} / \mathrm{m}^{2}$ ). In accordance with the American Society of Anesthesiologists Physical Status (ASA-PS) scale, 51 cases were classified as grade II and 43 cases were classified as grade III. Chronic diseases, namely, diabetes mellitus (DM) and hypertension (HT), were also evaluated; 14 patients had DM, and 59 had HT. This retrospective study was approved by the Kyushu Central Hospital review board (approval number: 21-1).

2.2. Laboratory Data Analysis. Blood examinations were performed on postoperative day (POD) 1 and 7. We used the neutrophil lymphocyte ratio (NLR) and monocyte lymphocyte ratio (MLR) to evaluate postoperative recovery. The association of postoperative NLR and MLR were clarified with the presence of osteoporosis. First, to confirm our hypothesis, we divided the 94 patients into two groups according to a YAM cutoff of $70 \%$. In addition, we divided 77 patients with a YAM $<70 \%$ into two groups according to a YAM cutoff of $50 \%$ (the median value among the 77 patients) and compared NLR and MLR. We then created a receiver operating characteristic (ROC) curve to predict which laboratory data were strongly correlated with YAM.

2.3. Statistical Analysis. All data were expressed as median and $25 \%-75 \%$ interquartile range (IQR). Differences between groups were evaluated using Fisher's exact test or Pearson's chi-square test. Nonnormally distributed variables were evaluated using the independent Wilcoxon signed-rank test. A $p$ value of $<0.05$ was considered statistically significant. Data analyses were performed using the JMP statistical software package (ver. 15; SAS Institute, Cary, NC).

\section{Results}

The patients' demographic data are summarized in Table 1. The BMI in patients with YAM $>70 \%$ was significantly
TABLE 1: Clinical parameters.

\begin{tabular}{lccc}
\hline Variable & $\begin{array}{c}\text { YAM } \geq 70 \% \\
(n=17)\end{array}$ & $\begin{array}{c}\text { YAM }<70 \% \\
(n=77)\end{array}$ & $P$ value \\
\hline Gender & $2 / 15$ & $16 / 61$ & 0.51 \\
male/female & $84(73-89.5)$ & $88(81.5-92)$ & 0.18 \\
Age (years) & $21.9(20-23.6)$ & $19.5(17.5-21.9)$ & $\mathbf{0 . 0 0 6}$ \\
BMI & & & \\
ASA-PS & 8 & 44 & 0.45 \\
2 & 9 & 33 & \\
3 & & & \\
Type of fracture & 9 & 40 & 0.94 \\
$\quad$ Neck & 8 & 37 & \\
$\quad$ Trochanteric & & & \\
Surgery & 5 & 9 & 0.58 \\
$\quad$ BHA & 12 & 68 & \\
$\quad$ ORIF & $6 / 22$ & $2 / 22$ & 0.12 \\
DM \pm & $10 / 7$ & $49 / 28$ & 0.71 \\
HT \pm & & &
\end{tabular}

ASA-PS: American Society of Anesthesiologists physical status, BHA: bipolar hip arthroplasty, BMI: body mass index, DM: diabetes mellitus, HT: hypertension, $n$ : number of patients, ORIF: open reduction and internal fixation, YAM: young adult mean.

higher than that in the YAM $<70 \%$ group (21.9 vs. 19.5 , respectively; $p=0.006$ ). There were no significant differences in any of the other clinical parameters between the two groups, namely, sex, age, ASA-PS, type of fracture, surgical procedures, and medical history.

The comparison of NLR and MLR according to a YAM cutoff of $70 \%$ is shown in Figure 1. On POD 1, patients with YAM $>70 \%$ had a median NLR of 5.7 (4.7-6.4) and a median MLR of 0.66 (0.51-0.82), which were significantly lower than for patients with YAM $<70 \%$ (5.7 vs. 8.8, $p<0.001$ and 0.66 vs. $0.9, p=0.0075$, respectively). Similarly, on POD 7, patients with YAM $>70 \%$ had a median NLR of $2.0(1.7-2.9)$ and a median MLR of $0.31(0.25-0.39)$, which were significantly lower than for patients with YAM $<70 \%$ (2.0 vs $3.5, p=0.001$ and 0.31 vs $0.43, p=0.0005$, respectively).

The comparison of NLR and MLR according to a YAM cutoff of $50 \%$ is shown in Figure 2. On POD 1, patients with YAM $<50 \%$ had a median NLR of $8.1(6.8-12.4)$ and MLR of $0.86(0.58-1.37)$. Patients with YAM ranging from $50 \%$ to $70 \%$ had a median NLR of 9.1 (6.7-11.0) and a median MLR of $0.99(0.63-1.18)$. There was no significant difference in both NLR and MLR between the two groups. Similarly, the data for POD 7 showed no difference between the two groups (NLR: 3.3 vs. 3.6 and LMR: 0.42 vs. 0.43 , respectively).

The results of the ROC curve analysis are shown in Figure 3. For NLR, data from POD 1 and POD 7 demonstrated that the values, 6.31 and 2.51, respectively, were predictive factors for YAM $<70 \%$ (sensitivity, 0.85; 1-specificity, 0.24 ; area under the curve, 0.81 vs. sensitivity, $0.81 ; 1$-specificity, 0.24 ; and area under the curve, 0.76 ). For MLR, data from POD 1 and POD 7 demonstrated that the values, 1.04 and 0.41 , respectively, were predictive factors for YAM $<70 \%$ (sensitivity, $0.45 ; 1$-specificity, 0.00 ; area under the curve, 0.71 vs. sensitivity, $0.62 ; 1-$ specificity, 0.18 ; and area under the curve, 0.77). 

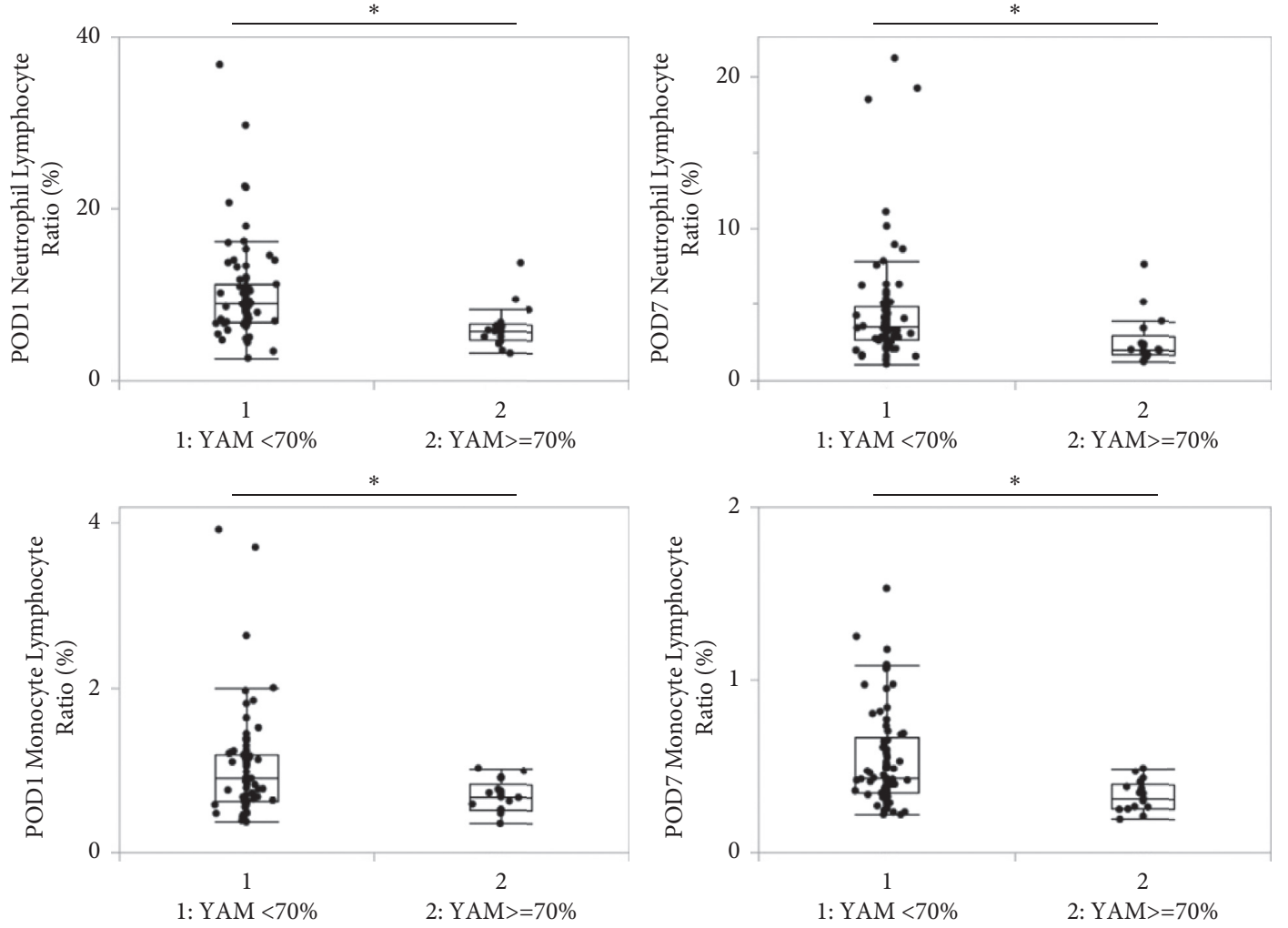

FIGURE 1: Comparison of neutrophil lymphocyte ratio (NLR) and monocyte lymphocyte ratio (MLR) according to a young adult mean (YAM) cutoff of $70 \%$. Patients with YAM $>70 \%$ had lower NLR and MLR values on postoperative day (POD) 1 and POD $7 .{ }^{*} P<0.01$.
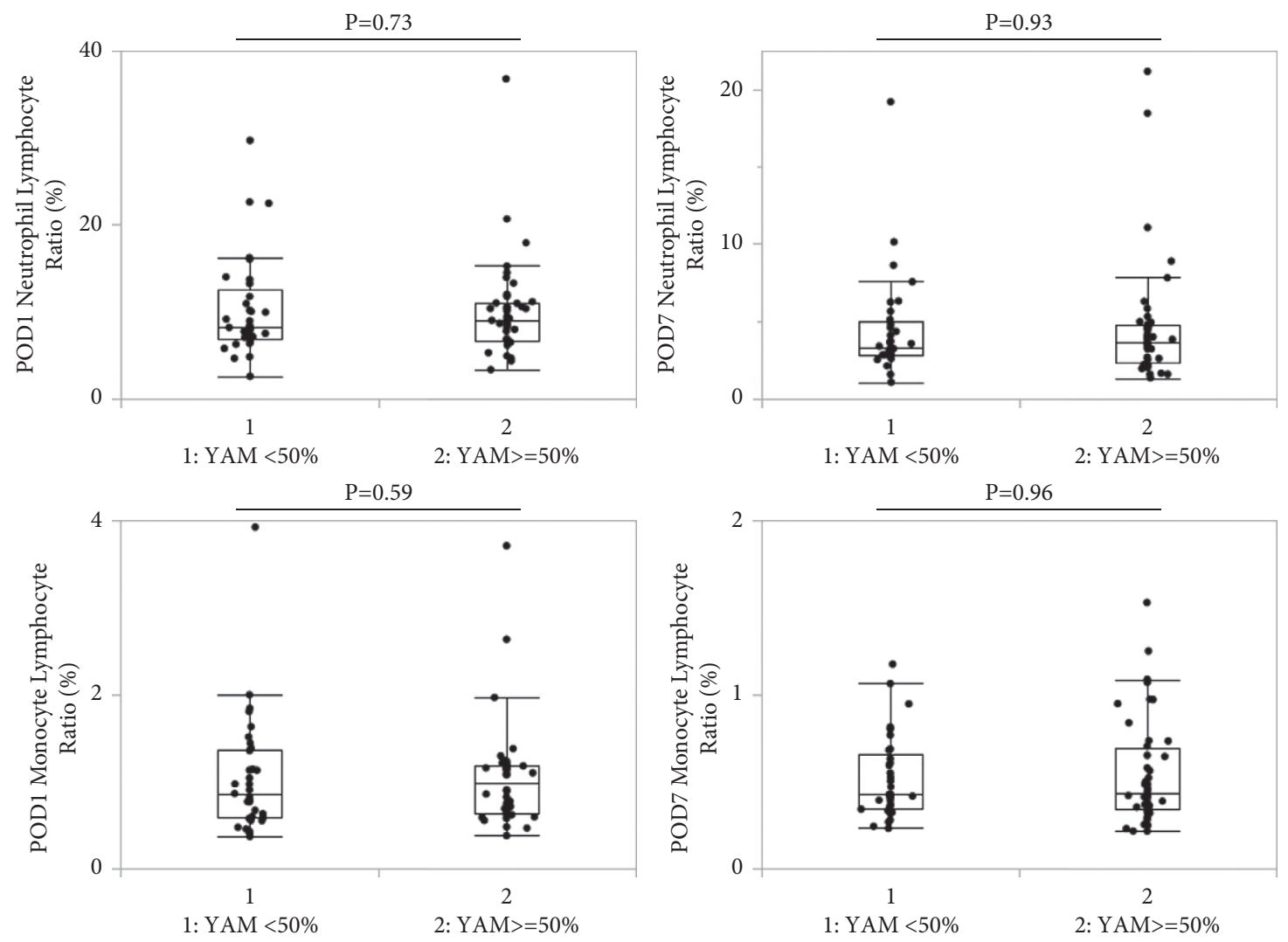

FIGURE 2: Comparison of neutrophil lymphocyte ratio (NLR) and monocyte lymphocyte ratio (MLR) according to a young adult mean (YAM) cutoff of 50\%. On postoperative day (POD) 1 and POD 7, there was no difference for both NLR and MLR between patients with YAM $>50 \%$ or $\mathrm{YAM}<50 \%$. 


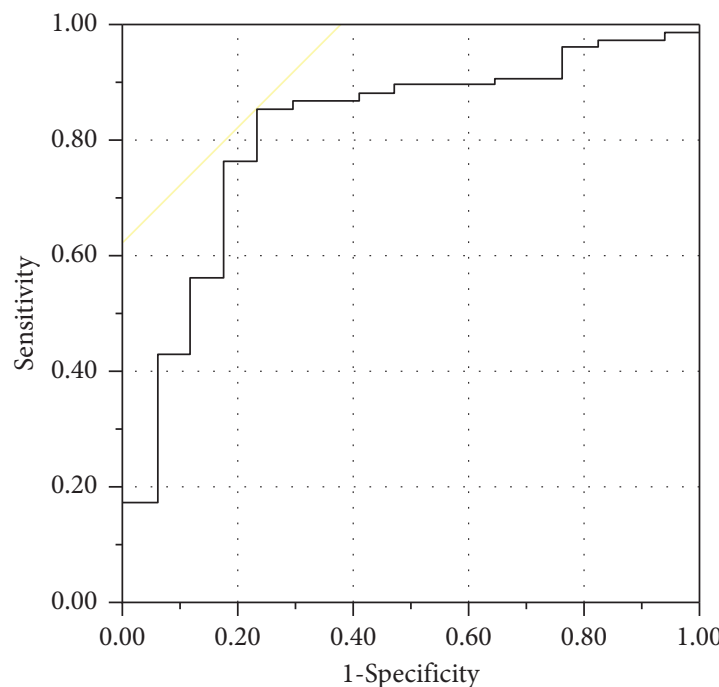

(a)

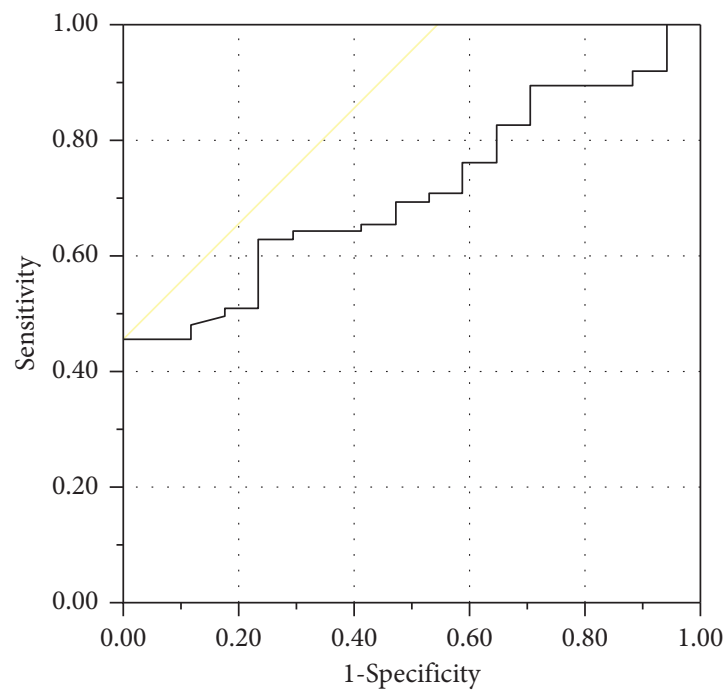

(c)

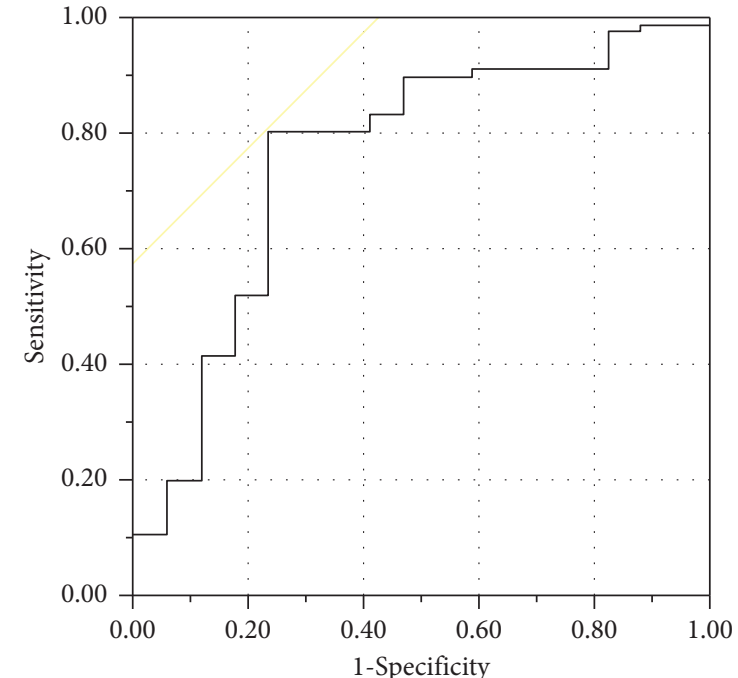

(b)

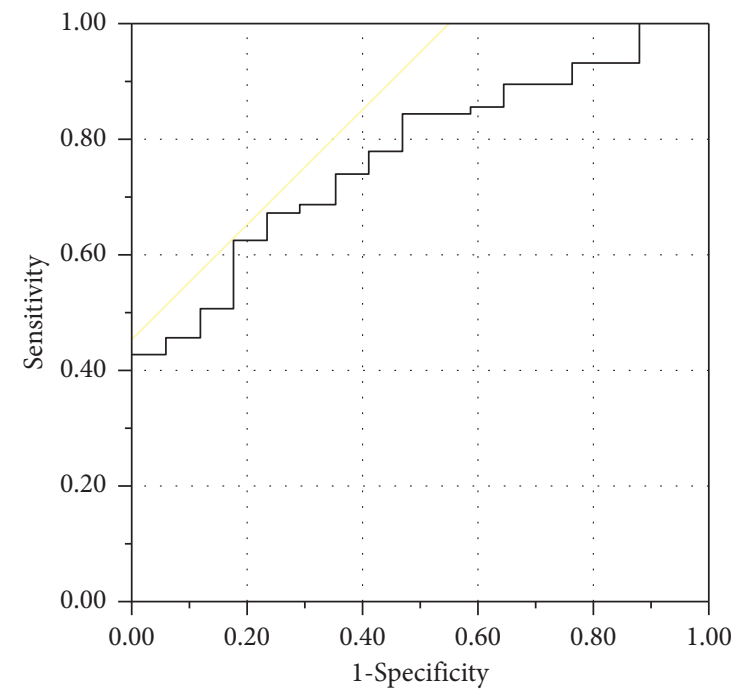

(d)

FIGURE 3: Receiver operating characteristic curves for neutrophil lymphocyte ratio (NLR) and monocyte lymphocyte ratio (MLR). (a) NLR of POD1. Sensitivity, 0.85; 1-specificity, 0.24; and area under the curve, 0.81. (b) NLR of POD7. Sensitivity, 0.81; 1 -specificity, 0.24; and area under the curve, 0.76. (c) MLR of POD1. Sensitivity, 0.45; 1-specificity, 0.00; and area under the curve, 0.71. (d) MLR of POD7. Sensitivity, 0.62 ; 1 -specificity, 0.18 ; and area under the curve, 0.77 .

\section{Discussion}

In this retrospective cohort study, we confirmed the hypothesis that a YAM cutoff of $<70 \%$ was appropriate regarding postoperative recovery in Japanese patients after hip fracture surgery. We used NLR and MLR as biomarkers for postoperative invasiveness. Patients with YAM $>70 \%$ had lower NLR and MLR values on both POD 1 and POD 7, suggesting that a YAM of $70 \%$ was an appropriate intervention threshold regarding postoperative recovery.

Hip fractures are a severe health problem in patients of advanced age because they can cause a significant decline in mobility and can decrease life expectancy [4]. Osteoporosis has a strong association with systemic metabolic disorders. Arterial calcification processes share common pathways with bone physiology, particularly osteoporosis [10]. Rhee et al. evaluated the relationship between receptor activator of nuclear factor-kappaB ligand (RANKL) gene polymorphism and aortic calcification [11]. Akune et al. showed that insulin receptor substrate signaling caused a potent bone anabolic action by insulin and insulin-like growth factor-I [12]. Furthermore, osteopontin, a phosphoglycoprotein relevant to the immune response, was upregulated in cases with low bone mineral density [13]. Considering these findings, intervention for osteoporosis is crucial to prevent fractures.

NLR and MLR have been studied and were found valuable for predicting the outcomes or prognosis of oncological diseases. Cook et al. found that an NLR value $\geq 9.3$ on the first POD following colorectal surgery was associated with increased mortality [14]. Yasui et al. reported that postoperative, but not preoperative, inflammation-based prognostic markers more accurately predicted prognosis in 
patients with colorectal cancer [15]. In orthopedics, an increased NLR after hip fracture operations was a risk factor for postoperative mortality $[16,17]$. MLR has a higher diagnostic value for osteoporosis [18]. We postulated that patients with YAM $<70 \%$ had higher inflammatory biomarker concentrations owing to the greater stress they sustained from surgery compared with patients with YAM $>70 \%$. As a result, our outcomes showed that patients with YAM $>70 \%$ had lower NLR and MLR values on both POD 1 and POD 7. There was no correlation between the laboratory data and YAM in patients with YAM $<70 \%$. The results of the ROC curve analysis demonstrated that both NLR and MLR on both POD 1 and POD 7 showed moderate accuracy. In particular, the specificity of MLR on POD 1 was quite high. These results suggested that YAM $>70 \%$ was an appropriate intervention threshold.

Several elements may be relevant to postoperative recovery after hip fracture surgery. In the current study, low BMI was associated with BMD $<70 \%$. Reginster et al. reviewed the link between osteoporosis and sarcopenia [19], and age-related loss of skeletal muscle was associated with cardiovascular mortality in another study [20]. Treatment to increase muscle volume in addition to BMD may help decrease surgical invasiveness and patients' subsequent healthy life expectancy.

\section{Conclusions}

The diagnosis of osteoporosis is based on bone mineral density measurements expressed as a percentage of the young adult mean (YAM) in Japan. Osteoporosis is defined as YAM $<70 \%$. Because osteoporosis has a strong association with systemic metabolic disorders, we postulated that patients with YAM $<70 \%$ had higher inflammatory biomarker concentrations owing to the higher systemic stress compared with YAM $>70 \%$. Patients with YAM $>70 \%$ had lower NLR and MLR values on POD 1 and POD 7 after hip surgery. YAM cutoff of $<70 \%$ was an appropriate intervention threshold regarding postoperative recovery after hip fracture surgery.

\section{Data Availability}

The data that support the findings of this study are available from the corresponding author.

\section{Conflicts of Interest}

The authors declare no conflicts of interest.

\section{Acknowledgments}

The authors thank Jane Charbonneau, DVM, from Edanz Group (https://en-author-services.edanz.com/ac) for editing a draft of this manuscript.

\section{References}

[1] H. Orimo, Y. Hayashi, M. Fukunaga et al., "Diagnostic criteria for primary osteoporosis: year 2000 revision," Journal of Bone and Mineral Metabolism, vol. 19, no. 6, pp. 331-337, 2001.
[2] S. Soen, M. Fukunaga, M. Fukunaga et al., "Diagnostic criteria for primary osteoporosis: year 2012 revision," Journal of Bone and Mineral Metabolism, vol. 31, no. 3, pp. 247-257, 2013.

[3] J. A. Kanis, H. Johansson, A. Odén, and E. V. McCloskey, "The distribution of FRAX-based probabilities in women from Japan," Journal of Bone and Mineral Metabolism, vol. 30, no. 6, pp. 700-705, 2012.

[4] H. Hagino, M. Osaki, R. Okuda, S. Enokida, and H. Nagashima, "Recent trends in the incidence of hip fracture in Tottori Prefecture, Japan: changes over 32 years," Archives of Osteoporosis, vol. 15, no. 1, p. 152, 2020.

[5] M. Wiens, M. Etminan, S. S. Gill, and B. Takkouche, "Effects of antihypertensive drug treatments on fracture outcomes: a meta-analysis of observational studies," Journal of Internal Medicine, vol. 260, no. 4, pp. 350-362, 2006.

[6] I. Kanazawa, T. Yamaguchi, M. Yamauchi et al., "Adiponectin is associated with changes in bone markers during glycemic control in type 2 diabetes mellitus," Journal of Clinical Endocrinology and Metabolism, vol. 94, no. 8, pp. 3031-3037, 2009.

[7] D. D. Sin, J. P. Man, and S. F. P. Man, “The risk of osteoporosis in Caucasian men and women with obstructive airways disease," The American Journal of Medicine, vol. 114, no. 1, pp. 10-14, 2003.

[8] K. E. Ensrud, L. Y. Lui, B. C. Taylor et al., "Renal function and risk of hip and vertebral fractures in older women," Archives of Internal Medicine, vol. 167, no. 2, pp. 133-139, 2007.

[9] T. V. Nguyen, J. R. Center, and J. A. Eisman, "Femoral neck bone loss predicts fracture risk independent of baseline BMD," Journal of Bone and Mineral Research, vol. 20, no. 7, pp. 1195-1201, 2005.

[10] B. Thompson and D. A. Towler, "Arterial calcification and bone physiology: role of the bone-vascular axis," Nature Reviews Endocrinology, vol. 8, no. 9, pp. 529-543, 2012.

[11] E.-J. Rhee, E.-J. Yun, K.-W. Oh et al., "The relationship between Receptor Activator of Nuclear Factor-.KAPPA.B Ligand (RANKL) gene polymorphism and aortic calcification in Korean women," Endocrine Journal, vol. 57, no. 6, pp. 541-549, 2010.

[12] T. Akune, N. Ogata, K. Hoshi et al., "Insulin receptor substrate- 2 maintains predominance of anabolic function over catabolic function of osteoblasts," Journal of Cell Biology, vol. 159, no. 1, pp. 147-156, 2002.

[13] V. C. Souza, W. M. Freitas, L. A. Quaglia et al., "Osteopontin in bone mineral density of very old Brazilians," Journal of Bone and Mineral Metabolism, vol. 31, no. 4, pp. 449-454, 2013.

[14] E. J. Cook, S. R. Walsh, N. Farooq, J. C. Alberts, T. A. Justin, and N. J. Keeling, "Post-operative neutrophil-lymphocyte ratio predicts complications following colorectal surgery," International Journal of Surgery, vol. 5, no. 1, pp. 27-30, 2007.

[15] K. Yasui, D. Shida, Y. Nakamura, Y. Ahiko, S. Tsukamoto, and Y. Kanemitsu, "Postoperative, but not preoperative, inflammation-based prognostic markers are prognostic factors in stage III colorectal cancer patients," British Journal of Cancer, vol. 124, no. 5, pp. 933-941, 2021.

[16] P. Forget, N. Moreau, H. Engel et al., "The neutrophil-tolymphocyte ratio (NLR) after surgery for hip fracture (HF)," Archives of Gerontology and Geriatrics, vol. 60, no. 2, pp. 366-371, 2015.

[17] A. Atlas, E. Duran, B. Pehlivan, V. F. Pehlivan, M. K. Erol, and N. Altay, "The effect of increased neutrophil lymphocyte ratio on mortality in patients operated on due to hip fracture," Cureus, vol. 12, no. 1, Article ID e6543, 2020. 
[18] K. Gao, W. Zhu, W. Liu et al., "The predictive role of monocyte-to-lymphocyte ratio in osteoporosis patient," Medicine, vol. 98, no. 34, Article ID e16793, 2019.

[19] J.-Y. Reginster, C. Beaudart, F. Buckinx, and O. Bruyère, "Osteoporosis and sarcopenia," Current Opinion in Clinical Nutrition and Metabolic Care, vol. 19, no. 1, pp. 31-36, 2016.

[20] A. J. Rodríguez, D. Scott, B. Khan et al., "Low relative lean mass is associated with increased likelihood of abdominal aortic calcification in community-dwelling older Australians," Calcified Tissue International, vol. 99, no. 4, pp. 340-349, 2016. 